\title{
Los valores compartidos: una reinterpretación política del confucianismo en Singapur
}

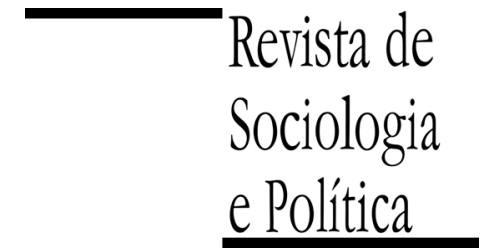

DOI 10.1590/1678-987314225102

\author{
Manuel de Jesús Rocha-Pino
}

\section{RESUMEN}

\begin{abstract}
Esta investigación estudia el proceso político realizado por el régimen del Partido de Acción Popular (PAP) de Singapur durante los años ochenta y noventa del siglo pasado para elaborar un proyecto de ideología nacional con el fin de legitimar al sistema político conformado en ese país a partir de su independencia. Este proyecto de ideología nacional denominado como "Valores Compartidos" tiene como uno de sus principales referentes a una versión del confucianismo chino la cual ha sido adaptada (y descontextualizada) por el régimen de Singapur para cumplir fines concretos, como el mantener la estabilidad de su sistema político y la de sus elites de gobierno. En la investigación se analiza como el proyecto de ideología nacional fue relacionado con el discurso de los "valores asiáticos" existente en la región de Asia del Este con la finalidad de consolidar el orden, el progreso y la identidad nacional en un país caracterizado por su diversidad multicultural. Como conclusión, la investigación ofrece que la incorporación de los "valores asiáticos" en el discurso oficial del régimen de Singapur se debió igualmente a la condición poscolonial de este país siendo una respuesta ideológica a la ideas orientalistas que se han generado sobre las viejas regiones coloniales de Asia desde el siglo XIX. EI estudio del discurso sobre los valores asiáticos en Asia del Este (en este caso, las particularidades que asumió en Singapur) durante los años ochenta y noventa puede contribuir a la investigación sobre el desarrollo histórico contemporáneo de esa región.
\end{abstract}

PALABRAS CLAVE: Partido de Acción Popular; valores asiáticos; Junzi; confucianismo; occidentalismo.

Recebido em 17 de Dezembro de 2012. Aprovado em 13 de Maio de 2013.

\section{Introducción ${ }^{1}$}

\footnotetext{
1 El autor agradece las sugerencias y comentarios de los tres dictaminadores anónimos de la Revista de Sociologia e Política.

2 Desde 1959 tres miembros del PAP han ocupado el cargo de Primer Ministro de Singapur: Lee Kuan Yew (1959-1990), Goh Chok Tong (1990-2004) y Lee Hsien Loong (desde 2004) quien es hijo de Lee Kuan Yew. Lee ha recibido los nombramientos de Ministro Senior (1990-2004) y Ministro Mentor de Singapur (desde 2004).
}

\begin{abstract}
partir de la década de los años ochenta del siglo pasado, el gobierno de Singapur comenzó a elaborar un proyecto de ideología nacional cen1 trado en el rechazo a lo que oficialmente identificó como "valores occidentales". Cabe resaltar que el régimen político en Singapur ha sido encabezado por el Partido de Acción Popular (People's Action Party, PAP) desde la independencia de ese país en $1959^{2}$.
\end{abstract}

En el presente trabajo se describe como dicho proyecto de ideología nacional eligió como uno de sus referentes principales a una reinterpretación del confucianismo, la cual fue adaptada por el discurso oficial del régimen del PAP de Singapur para cumplir ciertos fines concretos: entre otros fines instrumentales, mantener la estabilidad del sistema político y el continuo acceso al poder de sus élites partidistas en el gobierno de Singapur.

La invención de esta ideología nacional aspiraba a constituir un conjunto de pautas de conducta en los ámbitos de la moral y la esfera cívica y el régimen de Singapur la identificó con el concepto de "valores asiáticos". Dichos "valores asiáticos" perseguían consolidar el orden, el progreso y la identidad nacional en un país caracterizado por una diversidad multicultural herencia de su pasado colonial.

En contraposición, los así llamados "valores occidentales" se convirtieron en la representación de un imperialismo económico y cultural, continuador del régimen colonial que padeció Singapur desde el siglo XIX. Los "valores 
occidentales" que criticaba el régimen de Singapur eran básicamente de naturaleza moral como el individualismo, el hedonismo o la falta de respeto a ciertas conductas cívicas y a la autoridad de las personas mayores de edad.

El caso de Singapur puede considerarse un ejemplo de cómo la universalidad de un conjunto de valores, que pueden ser identificados con el liberalismo occidental, se ha encontrado en entredicho desde que algunos países ubicados en Asia del Este y el Sureste de Asia comenzaron a visualizar en sus propios valores tradicionales la causa y justificación de su progreso económico. Un proceso que además coincidió con el contexto de la Posguerra Fría.

Sin embargo, la reinterpretación e incluso reinvención de dichos valores tradicionales, en el caso del régimen de Singapur ciertos elementos del confucianismo, puede resultar igualmente un hecho propio de la modernidad. Es decir, algunas de estas tradiciones en realidad son el resultado de una historia reciente, en tanto que son readaptaciones de ciertos fragmentos de una cultura, considerada como tradicional, al cambio social en el contexto poscolonial.

El proceso anterior también estaría determinado por la condición poscolonial de Singapur: las razones que llevaron al PAP a asumir el confucianismo como una referencia para la articulación de su proyecto de ideología nacional residen en las características del desarrollo histórico de ese país.

En la investigación se analizará el documento político que sirvió de base al régimen del Partido de Acción Popular de Singapur en su proyecto para conformar una ideología nacional durante las décadas de los años ochenta y noventa del siglo XX: el documento sobre Valores Compartidos (Shared Values), aprobado por el Parlamento de Singapur en 1991. El producto ideológico más relevante de dicho proceso fue la identificación del proyecto de Valores Compartidos en el discurso de los valores asiáticos, primordialmente durante los años noventa. En el trabajo se describirán las repercusiones internas en la instrumentalización del proyecto de ideología nacional, así como las aspiraciones del régimen del PAP en una eventual internacionalización de su particular ideología sobre los "valores asiáticos" hasta el início de la crisis económica-financiera que abatió al Sureste de Asia entre 1997-1999.

\section{Las características del régimen político en Singapur}

Originalmente, la isla de Singapur fue un territorio habitado por población malaya mientras que los colonizadores británicos se apoyaron en una minoría de inmigrantes provenientes de China para realizar las labores administrativas del lugar. Con el tiempo, el favoritismo hacia la población china provocó oleadas continuas de inmigración de esa población a la isla.

Como territorio colonial, Singapur fue administrado mediante distintos regímenes británicos a partir de 1826 hasta que en 1942, durante la Segunda Guerra Mundial, fue declarado capital de los asentamientos (settlements) británicos en Malasia. Ya desde 1931 los inmigrantes chinos constituían el 74.3\% de la población de Singapur.

En el contexto de la invasión japonesa a Singapur en 1942 la isla fue designada capital de la región suroriental de la Gran Esfera de Co-prosperidad y fue rebautizada como Syonan-to o Luz de la isla del Sur. Tras la liberación aliada en 1945, Singapur quedó bajo una administración militar británica, como colonia separada de Malasia, con el fin de enfatizar la importancia estratégica del territorio adyacente al Estrecho de Malaca. Por su parte, la población malaya de Singapur se sintió más afín hacia la incorporación de Singapur a Malasia que a tener una vida independiente. 
$3 \mathrm{Al}$ obtener su independencia en 1959, Singapur pasó a formar parte de la Federación de Malasia. Sin embargo, la divergencia entre los proyectos de país entre la Federación, de población malaya mayoritariamente rural, y los intereses de la población urbana y mayoritariamente china de Singapur, provocaron la expulsión de la isla del seno de la Federación en agosto de 1965 (Chew 1991, p. 363).
Al reforzamiento de la identidad malaya en pos de la independencia ocurrió un declive en la migración china e india; sin embargo, la población china permaneció en Singapur como el grupo étnico económicamente más poderoso, con un mayor nivel de educación, un buen porcentaje de hablantes anglófonos y un mayor conocimiento sobre las labores de administración de la isla.

Debido a las características anteriores, la composición de Singapur causaba cierto malestar a los vecinos Malasia e Indonesia, quienes veían con desconfianza a la población china. Por ello, cuando la independencia le fue otorgada a la Federación de Malasia en 1957, fueron justamente los malayos quienes crearon una serie de barreras en el proceso de la integración de Singapur a la Federación. La consecuencia de los desacuerdos provocó la expulsión formal de Singapur del seno de la Federación de Malasia en 1965 (Chew 1991, p. 363) ${ }^{3}$. Como resultado del proceso colonial mencionado, Singapur es en la actualidad un país multiétnico con una mayoría de población china rodeado por países de población malaya - como la Federación de Malasia e Indonesia.

De la misma manera, debido su condición poscolonial, durante el proceso de conformación de una ideología nacional el régimen del PAP tendió a orientarse hacia una tradición de pensamiento relacionado con la etnia predominante para articular los principios ideológicos que otorgaran un fundamento a la integración de la nación: la tradición de pensamiento elegida durante los años ochenta y noventa del siglo XX fue el confucianismo, claramente asociada con la mayoría china ${ }^{4}$. Sin embargo, tal proceso fue llevado a cabo de manera contigua a un necesario discurso oficial sobre la condición multiétnica del país, esgrimido por el régimen, con el fin de crear un consenso sobre la unidad nacional entre todos los grupos étnicos habitantes en Singapur y así evitar tensiones que pudieran causar serios problemas sociales (como los disturbios raciales que habían ocurrido en 1964).

\section{II.1. La reforma política contenida en los Valores Compartidos}

${ }^{4}$ En 1990 la población de Singapur se conformaba de la siguiente forma: un $77.7 \%$ de la población era de origen chino, $14.1 \%$ de malayos, $7.1 \%$ de indios (tamiles y sikhs, entre otros) y $1.1 \%$ pertenecía al grupo denominado como "otros" (primordialmente "euroasiáticos", es decir, mestizos) (Tamney 1996, p. 1).

\footnotetext{
5 El PAP puede ser identificado con la definición ideal de Giovanni Sartori de un partido político hegemónico: un régimen en donde un partido domina al sistema político, controla el
}

Durante los años posteriores a su independencia, Singapur desarrolló un régimen de gobierno que, sin ser dictatorial, se orientó a ejercer un alto grado de intervencionismo gubernamental en la vida de sus habitantes. Dicha intervención fue instrumentada a través de un conjunto de políticas sociales como el acceso a la vivienda pública, la política de distribución de recursos hacia los diversos grupos étnicos o la educación pública y los valores políticos que serían interiorizados desde el sistema educativo. Las políticas públicas se articularon de tal modo que la población fuera dependiente de los recursos estatales y se justificaron en base a criterios como: la preservación del orden y la seguridad nacional; el aumento del nivel de vida de la población y la continuación del éxito económico del país; el rechazo a ciertos elementos culturales "indeseables" que eran considerados como "provenientes" del Occidente y la preservación de los valores tradicionales que servirían como guías de la nación. Los anteriores criterios definieron, de la misma manera, las formas de participación política de los ciudadanos.

En este sentido el sistema político de Singapur puede ser definido como autoritario, ya que si bien posee ciertas instituciones como un Parlamento y un sistema electoral basado en la democracia representativa, el régimen de gobierno es controlado por el PAP en tanto partido hegemónico 5 . De la misma forma el régimen ha impulsado una organización social cuyos valores se orientan a la imposición de los intereses grupales sobre los individuales, fortaleciendo una relación paternalista con la población. A través de esta relación se persuade a la sociedad de Singapur sobre la necesidad de la continuidad del liderazgo del PAP y sus élites para mantener el progreso y la unidad nacional. 
acceso a los cargos y recursos públicos y puede permitir la existencia de otros partidos políticos "periféricos" o "secundarios" que no signifiquen una oposición real en los procesos electorales (Sartori 2005, pp. 204-211).
A su vez, la formación del Estado, preocupado por el bienestar social, se ha centrado simultáneamente en las relaciones de dependencia entre el gobierno y las empresas privadas de Singapur para instrumentar las políticas de desarrollo, mantener la gobernabilidad y la orientación de libre mercado del sistema económico. Cabe resaltar que los miembros de la élite política del PAP suelen realizar su formación profesional en las grandes empresas singapurenses, en las que se preparan para la vida política. Como comenta el sociólogo Chua Beng Huat:

"Una nación requiere una cultura nacional para definir sus fronteras geográficas. Esto es una necesidad en Singapur porque en las pasadas tres décadas de historia nacional, las energías de la población se enfocaron hacia la construcción de la economía nacional" (Chua 1995, p. 14).

Uno de los principales retos del régimen de Singapur ha residido en generar el discurso ideológico adecuado, que legitime y otorgue un contenido moral a las políticas y hacerles alcanzar un alto nivel de aceptación social.

A finales de los años ochenta, el PAP inició un proyecto para la construcción de una ideología nacional apropiándose de algunos elementos de la tradición confuciana para así emitir cierta coherencia discursiva entre el orden moral que buscaba establecer y las condiciones de orden necesarias para proseguir con el modelo de crecimiento económico e industrial y su desarrollo institucional.

Los principales elementos que buscaba consolidar esta ideología eran: justificar la existencia de ciertas relaciones jerárquicas para la preservación del orden; la alta apreciación del trabajo duro y la educación; la importancia de la familia como parte fundamental de la sociedad; la frugalidad; el respeto a las decisiones de la autoridad; y la valoración de la 'meritocracia' como el criterio básico de la movilidad social. Uno de los objetivos básicos era reformar a las instituciones públicas y así instrumentar las políticas que materializaran institucionalmente los contenidos de la ideología nacional en formación, estableciendo una doble función entre mantener el nivel de vida de la población mientras se acrecentaba la dependencia de esta sociedad hacia las políticas del régimen. Cabe establecer que dichos valores cívicos no tenían un trasfondo religioso sino que se podían “...expresar en el proceso de racionalización de la vida cotidiana” (Alatas 2002, p. 115).

Es relevante notar que si bien los valores confucianos tradicionales se relacionan inmediatamente con la mayoría china, el gobierno fue muy cuidadoso de que su presencia (incorporada en el discurso nacional) no se interpretara como un intento de establecer una hegemonía cultural china sobre el resto de las minorías que conviven en Singapur, como la malaya o la tamil (originaria del sur de la India).

$\mathrm{Al}$ promover la igualdad entre los grupos étnicos, el régimen asumió un espacio neutral, convirtiéndose en un garante de los intereses de todos los grupos "raciales". Esta posición neutral se institucionalizó en 1990 por medio de un Acta para el Mantenimiento de la Armonía Racial - el discurso oficial del régimen equiparó el término "raza" con el de etnia (Chua 1995, p. 9).

La ideología nacional buscaba incluir la promesa oficial de que el éxito del país sería compartido de manera equitativa, siempre y cuando no se perdiera el orden institucional y la armonía social. En la búsqueda de esta armonía también se apeló al sacrificio individual, el trabajo duro y la formación del consenso necesario para la realización del proyecto de nación. De la misma forma, la necesidad de constituir cierto pensamiento propio de Singapur con el fin de establecer una ideología nacional intentó contrarrestar los peligros que, de acuerdo a los intereses del régimen, entrañaba (y sigue entrañando) la internacionalización del país y así formular una política exterior independiente. 
En 1988, el entonces Vice Primer Ministro de Singapur Goh Chok Tong (quien sucedería a Lee Kuan Yew en el cargo como Primer Ministro en 1990) sugirió que debían iniciarse los trabajos para desarrollar la ideología nacional alrededor de la cual se integrarían todos los habitantes del país sin importar etnia o credo. Después de un par de años de debates, en 1991 fue presentado el proyecto sobre la ideología nacional al Parlamento de Singapur para su aprobación: el proyecto oficial tenía el título de Shared Values (Valores Compartidos). El propósito del documento era "consolidar y desarrollar una identidad de Singapur" al adoptar en un proyecto de ideología nacional algunos elementos de la herencia cultural, las actitudes morales y los valores cívicos del país (Singapore Government 1991, p. 1). Este documento contenía los elementos que, de acuerdo al discurso oficial, lo hacían compatible con el confucianismo (Kuo 1996, pp. 308-309).

Una vez aprobado el proyecto constitucional, el gobierno de Singapur publicó el documento sobre Valores Compartidos resaltando los cinco principios que sustentarían a la ideología nacional:

a) la nación debe estar por encima de la comunidad y la sociedad antes del individuo: poner los intereses de la sociedad por delante de lo individual;

b) la familia es la unidad básica de la sociedad;

c) buscar la solución de los problemas a través de consensos y no de imposiciones;

d) promover la armonía y la tolerancia entre las diferentes religiones y etnias;

e) respeto y apoyo de la comunidad al individuo (Singapore Government 1991, p. 1).

En una declaración realizada en 1991, Lee Kuan Yew prescribió:

"Goh Chok Tong y su equipo han invertido mucho tiempo y esfuerzo en obtener una mayoría de acuerdo sobre nuestros cinco valores compartidos. Pero recuerdo que esos cinco valores no hacen una filosofía de vida completa. Los chinos deben profundizar en sus valores tradicionales del confucianismo, el taoismo y el folklore chino para completarlos. Los malayos deben basarse en las costumbres malayas y en el islam, los indios en sus costumbres y en la religión hindú. Si nosotros intentamos poner todas esas tradiciones en un solo sitio, obtendremos una mezcla indescriptible" (Lee 1991, p. 25) ${ }^{6}$.

de febrero de 1991

A su vez Valores Compartidos se presentaba como un proyecto a largo plazo.

"Si la presente generación no guía a las nuevas generaciones, quien sabe qué nuevos valores adoptarán. Estas pueden perder, de manera inadvertida, sus costumbres o los valores que han apuntalado el éxito de Singapur" (Singapore Government 1991, p. 2).

Ciertamente a principios de los años ochenta la población de Singapur ya comenzaba a disfrutar de niveles de vida cada vez más altos, pero al mismo tiempo hubo incrementos en la incidencia de crímenes, divorcios, abortos y un cambio en la actitud de los grupos más jóvenes de la sociedad, los cuales fueron considerados "indeseablemente" individualistas. Este proceso de cambio social fue denunciado por el régimen del PAP como una "occidentalización" del país (Hill 1996, pp. 195-196).

Los Valores Compartidos de Singapur aspiraban a establecer una sociedad de rasgos diferentes a los occidentales y orientados particularmente hacia la "tradición" (Tamney 1996, p. 19). Como declaraba Goh Chok Tong en 1990, previamente a la oficialización de los Valores Compartidos: 
${ }^{7}$ Declaración realizada el 19 de diciembre de 1990.
"El sistema de valores que nosotros queremos es uno que asegurará a largo plazo la supervivencia de Singapur como nación. Este sistema de valores es la familia extendida, las relaciones basadas en la jerarquía social, el trabajo duro y el auto sacrificio" (Goh 1990, p. 27) .

Al mismo tiempo, los líderes del PAP exigieron al conjunto de los actores políticos de Singapur una moderación inmediata en sus demandas (los partidos políticos de la oposición fueron llamados a la contención política y en sus críticas a las instituciones) en el nombre del "interés nacional" (Chua 1993, p. 22). El consenso ideológico, desde la perspectiva de Chua, estableció las condiciones para que el grupo gobernante impusiera sus ideas a los gobernados, mientras que el público reproduciría dichas ideas "naturalmente" en la realidad cotidiana: los Valores Compartidos determinaron una ideología comunitaria que legitimara el consenso político (Chua 1995, p. 128).

A pesar de la realización de procesos electorales de representatividad democrática, durante los años ochenta la élite del PAP no buscó legitimar a su gobierno únicamente a través del sistema electoral y de partidos, sino mediante una declarada superioridad moral e intelectual de los dirigentes. "En 1984, el PAP experimentó con un elaborado proceso de escrutinio hacia sus miembros que incluyó exámenes de inteligencia y psicológicos" (Englehart 2000, p. 553). El mensaje al público era que el PAP estaba en la búsqueda de la mejor y más talentosa élite para gobernar. Los Valores Compartidos ofrecieron al régimen la posibilidad de elevar su elitista sistema de movilidad política al rango de la ideología nacional. Al descontextualizar al confucianismo y adoptarlo a la realidad del sistema político, la ideología nacional alcanzaba su fin instrumental, también legitimando al régimen mediante la efectividad de sus funcionarios: algo relevante al ser presentada, en el documento de Valores Compartidos, una interpretación sobre el concepto confuciano del gobernante virtuoso o Junzi.

\section{Elementos confucianos incorporados en los Valores Compartidos}

\section{III.1. Una breve definición del confucianismo y su ética}

${ }^{8}$ En el trabajo se ha usado el sistema de transliteración pinyin para los nombres y conceptos en idioma chino.

9 El Periodo de Primavera y Otoño (722-481 a.e.c) fue sucedido por el Periodo de los Estados Combatientes (403-221 a.e.c). Con la unificación de China en un solo Imperio por la Dinastía Qín en 221 a.e.c finaliza el periodo de la Dinastía Zhou.

${ }^{10}$ La época de Confucio atestiguó uno de los periodos
El confucianismo es la filosofía política y moral atribuida a Kong zi, o Confucio por su nombre latinizado, quien nació en el año 551 antes de la era común (a.e.c.) en la actual Provincia de Shandong en China ${ }^{8}$. Confucio se concebía a sí mismo como un educador y dedicó su vida a la enseñanza de un sistema ético basado en las viejas costumbres y rituales chinos anteriores a la Dinastía Zhou (1027-221 a.e.c), con el fin de enseñar a los gobernantes y al hombre común de su época los valores morales y cívicos que, en el pasado, habían estructurado una sociedad justa y armónica. El confucianismo fue la ideología oficial durante la mayor parte de duración del Imperio Chino tradicional (dividido en sus diferentes etapas dinásticas) hasta el final de la Dinastía Qing en 1912.

El ideal confuciano de una sociedad armónica, por desgracia, se había perdido debido a las ambiciones políticas y a las guerras provocadas por la turbulencia política durante el periodo en el que vivió Confucio, conocido como el Periodo de Primavera y Otoño ${ }^{9}$. A Confucio le correspondió una era violenta, pero su respuesta hacia ella fue la elaboración de un sistema ético que buscara la felicidad del hombre de acuerdo a lo que el filosofo consideró debía ser la armonía social $^{10}$. A su vez Confucio no se consideraba un innovador en algún sentido, para él su obra había consistido en volver a difundir las viejas ideas y rituales prevalecientes en un pasado ideal, anterior a la propia Dinastía Zhou, conocida como la Edad de Oro de China, concepto en el que se mezclan lo histórico y lo mítico. Las ideas confucianas, si bien no poseen una metafísica 
de cambio más trascendentales de la historia china tanto en comunicaciones, tecnología militar, formas de cultivo e ideas. Las ideas filosóficas que con el tiempo determinarán la cultura política tradicional china son producto de este periodo. Véase Allinson (1990, pp. 1-24) y Schwartz (1985, pp. 56-134).

${ }^{11}$ Las cinco virtudes que componen las bases de la ética del comportamiento confuciano son: la benevolencia (ren), la rectitud (yi), la propiedad ante los ritos(li), el conocimiento (zhi), y la integridad (xin). mediante la cual se busque la trascendencia del ser, buscan idealmente el retorno a aquel pasado mítico en donde todo era mejor que en el presente.

Como ocurre con la mayoría de los sabios antiguos, Confucio no dejó una obra íntegramente escrita por él, sino que sus trabajos han llegado a nosotros a través de los alumnos y comentaristas que Confucio ha tenido desde entonces. Difundido desde la muerte de Confucio en el siglo V a.e.c, el confucianismo (mejor conocido en su inicio como Rujia - o escuela de los letrados) fue dispersándose por todo el territorio chino y sus numerosos reinos hasta que fue adoptado como ideología del Estado en el siglo II a.e.c durante el periodo de la Dinastía Han: es decir, una vez que el país fue unificado bajo una misma dinastía gobernante. Desde este tiempo sus ideas mejor conocidas fueron enseñadas como parte de la ideología estatal a través de recopilaciones como el Lun Yu o Analectas (Fung 1998, p. 39).

Los elementos básicos del confucianismo son el énfasis en la educación de la población como forma de mantener la virtudes morales de la persona y con ello la armonía social; la selección de funcionarios a través de un sistema de exámenes civiles; el concepto de piedad filial según el cual los hijos deben guardar devoción hacia sus padres y antepasados, tanto en lo afectivo como en lo material; y un sistema jerárquico de ordenamiento social coherente en el que las relaciones familiares guardan una correspondencia con el sistema de autoridad del gobierno. "La visión confuciana se centra sobre la extensión de la conciencia de la persona a través de la familia a los grupos locales - y con ello hasta el Estado - haciendo que el mundo como un todo orgánico posea un carácter universal" (Zhang 1999, p. 3). Como afirma el clásico confuciano La Gran Sabiduría o Da Xue: "las personas cultivadas han regulado a sus familias. Cuando sus familias han sido reguladas, sus Estados fueron correctamente gobernados. Cuando sus Estados fueron correctamente gobernados, la totalidad del reino estuvo en paz y feliz". Este conjunto de axiomas es la guía del ordenamiento social de gobernantes y el pueblo chino por igual.

Para entender el proyecto de ideología nacional de Singapur se debe definir el ordenamiento moral que posee el confucianismo en tanto doctrina filosófica que se orienta a establecer los fundamentos de una ética social. La pertenencia del individuo a su grupo social está condicionada por el seguimiento puntual de las reglas civilizadas del comportamiento ético y el seguimiento de los rituales (li) ${ }^{11}$. El punto de partida del pensamiento político de esta doctrina moral ha sido denominado como "la rectificación de los nombres" o zhengming, concepto que significa, literalmente: "ajustar los poderes y deberes, derechos y obligaciones del gobernante y el ministro, superior e inferior, acordes con las instituciones prevalecientes en la China de los Zhou" (Hsiao 1979, p. 99). Es decir, las palabras gobernante y gobernado, padre e hijo asignan a cada individuo un lugar en la sociedad. La no aceptación de cada papel social que corresponde a cada miembro de la sociedad tiene como consecuencia el caos (ibidem). Por eso el filósofo debe mostrar los derechos y obligaciones de cada término: cuando un hombre realiza el papel que le corresponde entonces puede ser llamado con justicia padre, hijo, gobernante o ministro, etc. "El príncipe debe ser un príncipe, el ministro un ministro, el padre un padre y el hijo un hijo" (Confucio 1979, p. 118). La rectificación de los nombres representa el inicio del pensamiento político confuciano a su vez derivado de la filosofía moral:

"cada nombre contiene ciertas características que constituyen la esencia de aquélla clase de cosas para las cuáles dicho nombre se aplica. Las cosas, por ello, debían estar de acuerdo con dicha esencia ideal. La esencia del gobernante era lo que el gobernante idealmente debía de ser o, lo que en chino es llamado, la "forma del gobernante". Si el gobernante actúa de acuerdo con esta forma del gobernante, entonces él es un verdadero gobernante, tanto de hecho como de nombre. Debía existir una concordancia entre el nombre y lo fáctico. Cada 
${ }^{12}$ Confucio comenta en los Analectas: "Gobierna al pueblo por reglas, mantén el orden mediante castigos y él escapará de ti y perderás todo el auto respeto. Gobierna por medio de la fuerza de la moral, mantén el orden entre el pueblo por medio de los rituales y conservarás su auto respeto y el pueblo estará de acuerdo contigo en todo lo que hagas" (Confucio 1979, p. 63). nombre en las relaciones sociales implica ciertas responsabilidades y deberes. Gobernante, ministro, padre e hijo son, todos, los nombres de éstas relaciones sociales, y los individuos poseyendo esos nombres deben seguir sus responsabilidades y deberes acordemente" (Fung 1998, pp. 41-42).

La rectificación de los nombres demandaba la confianza por parte de todos los individuos de que cada uno realizaría su papel específico en la sociedad, por ello el orden confuciano confiaba más en la aceptación moral de sus normas que en la coerción legal para el mantenimiento del orden (Hsiao 1979, p. 101) ${ }^{12}$. Las responsabilidades y deberes de cada nombre le otorgaban a cada individuo el lugar social que le correspondía, así la rectificación de los nombres se convertía en un criterio básico para articular la estratificación social. Sin embargo, la sociedad china no era una sociedad de estamentos fijos (o de castas): el individuo siempre podía ascender socialmente (podía rectificar su nombre) mediante los méritos sociales que pudiera conseguir. El modo ideal en que el individuo confuciano podía abrirse las puertas de la movilidad social era mediante el estudio, el auto perfeccionamiento moral, el trabajo arduo y, durante la época imperial, una carrera burocrática. Los famosos exámenes de acceso al servicio civil, en los que se evaluaba el conocimiento de los clásicos confucianos de los futuros funcionarios, eran el modo más honorable para ascender en la escala social.

Dice Confucio a un gobernante de su época: "Gobernar significa rectificar. $\mathrm{Si}$ tú diriges al pueblo con rectitud, ¿quien estaría en contra de lo recto? [...] $\mathrm{Si}$ un hombre se esfuerza por hacerse a sí mismo correcto, ¿qué dificultad encontrará para tomar parte en el gobierno? Si él no puede hacerse a sí mismo correcto ¿qué asunto habrá él de hacer correcto para otros?" (Confucio 1979, p. 115 y 120).

Con esto Confucio nos quiere decir que el hombre superior, o Junzi, actúa conforme con lo que es correcto y que por ello el pueblo no será contrario a ser bien gobernado por él. Si el gobernante ama la rectitud y actúa conforme a las virtudes será un ejemplo para todos. El mantenimiento del orden era el fin del gobernante confuciano, a su vez esta armonía se conseguía básicamente por medio de tres criterios: garantizar la alimentación del pueblo, educar y gobernar: "Los instrumentos a través de los cuales se alimenta y se educa al pueblo son: la virtud así como los ritos, mientras que los instrumentos que sirven para gobernar son las políticas y los castigos. La virtud - el ejemplo que debe dar el gobernante por medio de su propio comportamiento - y los ritos eran los instrumentos principales mientras que los castigos y las políticas eran auxiliares. De esta forma, la política central que mas enfatizaba Confucio era la transformación de la sociedad a través de la educación" (Hsiao 1979, p. 109).

La educación buscaba la interiorización de los valores aceptados por la sociedad promovidos desde el Estado, de estos valores el principal era la benevolencia: "El hombre de benevolencia desea el bien para sí mismo y también el bien para los demás, al cultivarse a sí mismo, busca cultivar a los otros" (Confucio 1979, p. 85).

Como se mencionó el confucianismo no busca elaborar explicaciones metafísicas sobre el ser, ya sean teorías sobre la creación del mundo o sobre la creación del hombre. Las reglas de comportamiento en un mundo no creado por dioses o entes metafísicos radican en su aceptación o no por parte de los individuos, sin que alguna medida coercitiva fuera de la realidad los obligue a seguirlas. De la misma manera, la obligación a seguir las normas éticas confucianas radica en la aceptación o no del individuo por parte del resto de la sociedad.

Aquel que no se comportaba de acuerdo a las virtudes confucianas de benevolencia, reciprocidad, rectitud o el seguimiento de los ritos (es decir, el 
equivalente cívico confuciano de la ritualidad religiosa en otros sistemas de creencias), era inmediatamente rechazado por la sociedad. Al mismo tiempo, los individuos poseen el mandato interno de relacionarse con la sociedad de acuerdo al lugar que les corresponde. Este comportamiento se considera un deber y debe realizarse en la actuación constante del individuo: un Emperador tenía la obligación de comportarse con la benevolencia del Junzi, un letrado como un hombre ejemplar en su comportamiento dada su sabiduría sobre los clásicos confucianos, un soldado como lo que era, un campesino debía dotar de alimentos al Imperio y así contribuir a su prosperidad, etc.

El cumplimiento de las obligaciones de manera recíproca entre los miembros de la sociedad correspondía el concepto de reciprocidad el cual conforma la denominada Regla de Oro o ley de reciprocidad del confucianismo: "No hagas a otros lo que no deseas para ti mismo" (Bo 2004, p. 222):

"El concepto de shu en las Analectas se refiere al método de pensamiento moral. Más específicamente, el shù es una clase de pensamiento analógico: tomando a uno mismo como medida cuando uno decide de qué manera debería tratar a los demás. El shu podía tener un aspecto negativo y uno positivo: en el negativo la persona se tomaba como medida: lo que no haría nunca a los otros es lo que nunca desearía para sí mismo; en el aspecto positivo la persona haría a los demás lo mismo que desearía para sí mismo. [...] Confucio describe al shu como el método del ren. Mediante el shu el individuo podía identificarse con los otros y, de esa manera, tratar a los otros como a un yo hipotético. Por ello el shu podía capturar el elemento esencial del ren: la benevolencia" (Sin 2000, respectivamente, pp. 507-508 y 512).

\section{III.2. El proyecto de Valores Compartidos y el concepto de Junzi}

${ }_{13}$ Dicha discriminación evidenció el carácter instrumental de los elementos confucianos incorporados en el proyecto de Valores Compartidos. Previamente a los años noventa Singapur se había caracterizado por tener un conjunto de normas sumamente severas para reglamentar y castigar ciertas conductas incívicas como arrojar basura en la calle, mascar chicle en lugares públicos y el vandalismo.
Si bien el régimen del PAP no buscó revivir los aspectos ritualísticos del sistema de creencias confuciano, sí trató de integrar fragmentos de su visión de la ética en el proceso de articulación de un consenso sobre la identidad nacional de Singapur. El resultado de dicho consenso, a su vez, se podía elevar al rango de ideología nacional dado su carácter cívico secular. De la misma manera, otro elemento importante del confucianismo como la prevalencia de las normas morales por encima de las leyes escritas no fue tomado en cuenta en los planes del $\mathrm{PAP}^{13}$.

"El más conspicuo de esos elementos confucianos contenidos en el Documento sobre Valores Compartidos, aprobado por el Parlamento, fue el concepto del Junzi?, es decir, el gobierno del "rey virtuoso". Este concepto le permitía al PAP conservar su postura elitista y paternalista porque el partido podía sugerir que sus líderes eran de una clase de hombres virtuosos" (Kuo 1996, p. 308). De esa manera la élite de gobierno se auto identificaba con otro elemento clave del proyecto de ideología nacional al establecer los criterios básicos para la selección de funcionarios en su sistema meritocrático.

Valores Compartidos hacía las siguientes referencias al perfil del gobernante ideal:

"[parágrafo número] 41. Muchos ideales confucianos son relevantes para Singapur. Por ejemplo, la importancia de las relaciones humanas y colocar a la sociedad sobre el individuo son ideas clave de estos Valores Compartidos. El concepto del gobierno por un hombre honorable o Junzi, quien tiene la obligación de hacer un bien por el pueblo y quien tiene la confianza y el respeto de la población, se ajusta mejor entre nosotros que la idea occidental de que un gobierno debería tener poderes limitados y que debería ser tratado con sospecha hasta que se demuestre lo contrario" (Singapore Government 1991, p. 8).

La idea del Junzi o los criterios que le darían forma se encontraban presentes en las declaraciones de la élite de gobierno de Singapur, y era una de las ideas 
más recurrentes del Primer Ministro Lee Kuan Yew en ese momento. "En una sociedad dada", él dice,

"de mil bebés que nacen, hay varios cientos que se acercan a la categoría de genios y un porcentaje de bebes promedio. Lamento que constantemente esté preocupado por lo que harán quienes se encuentran por encima del promedio. Estoy convencido que ellos son quienes decidirán el porvenir. Son quienes estén por encima del promedio en donde cualquier sociedad basa su paz" (Lee Kuan Yew apud Chang 2003, p. 92).

Sin embargo, aunque la ideología beneficiaba a una élite cerrada, de acuerdo al discurso oficial toda la población se hacía partícipe de ella; como mencionaba el Ministro de Educación Goh Keng Swee en la década de los años ochenta: "El confucianismo en Singapur no será únicamente para los salones de clase. Este será interpretado como un código de conducta personal para el Singapur moderno y será promovido en la forma de un debate público y discutido en los medios de comunicación" (apud Englehart 2000, p. 557). Los Valores Compartidos resultaron inclusivos por lo menos en su sistema de obligaciones.

Otro elemento relevante en el proyecto de Valores Compartidos fue su énfasis en los valores de Singapur (ahora el confucianismo, reinterpretado por el régimen del PAP), diferenciándolos de los valores extranjeros u occidentales, algo necesario para reclamar la excepcionalidad del caso de Singapur.

De esta manera, en el proyecto de ideología nacional se hacía presente la tensión entre valores compartidos y valores occidentales como sustento de la legitimidad del régimen del PAP. Como menciona el propio Lee Kuan Yew:

"Un pueblo formado en los valores chinos tendría más disciplina, sería más cortés y más respetuoso con sus mayores. El resultado sería una sociedad más ordenada. Cuando esos valores fueran diluidos por una educación inglesa el resultado sería una sociedad menos vigorosa y disciplinada a causa de su comportamiento" (Lee 1998, p. 546).

En el documento de Valores Compartidos se hace referencia a esta distinción en los parágrafos 25 y 26.

"25. Ninguna sociedad asiática ha sido modelada sobre el prototipo occidental. Muchas lo han intentado y se encontraron con unas instituciones occidentales mal adaptadas a su temperamento nacional y a su situación internacional" (Singapore Government, 1991, p. 5).

De esta manera, el discurso oficial de Singapur rechazaba a los valores occidentales reivindicando su identidad asiática, para evitar que su población se volviera permeable ante la influencia de los valores "no asiáticos". Lo anterior conformó una especie de orientalismo desde Singapur hacia el conjunto de lo que se considera como "occidental".

"[Parágrafo número] 26. Singapur es una sociedad asiática. Esta siempre ha fortalecido los intereses de grupo de manera más enfática que los individuales. Ello ha fortalecido la cohesión social y ha permitido a los singapurenses trabajar juntos para vencer los problemas colectivamente y hacerlo más exitosamente que otras sociedades" (ibidem).

El rechazo a unos valores occidentales que distraían a la población de sus obligaciones ciudadanas se convirtió en uno de los elementos retóricos del PAP. Los valores occidentales fueron usados como un contraste frente los valores que, a juicio del régimen, se propagaban desde los medios de comunicación para modificar la estructura social del país (Englehart 2000, p. 562).

Inevitablemente la aparición de los Valores Compartidos provocaría ciertas tensiones en la sociedad de Singapur. Por esta razón, frente a la alta posibilidad de una oposición por parte de un porcentaje de la población china y la hostilidad de sectores de todas las minorías étnicas, el PAP advirtió en su discurso que el 
contenido confuciano de los Valores Compartidos no tenían un carácter que pudiera identificarse únicamente con el sector poblacional de origen chino:

\begin{abstract}
"[Parágrafo número] 39. Inicialmente los singapurenses no chinos estaban preocupados porque los Valores Compartidos pudieran convertirse en un subterfugio para la imposición de los valores sino-confucianos sobre ellos. Esto nunca fue la intención del gobierno. El gobierno nunca ha permitido que una mayoría racial se imponga a una comunidad minoritaria. No puede forzar al confucianismo sobre los no chinos o dejar a los Valores Compartidos encabezar un chovinismo chino" (Singapore Government 1991, p. 7).

"Los Valores Compartidos rechazaron la tendencia chovinista percibida en la campaña de los valores confucianos (asociada con la población china) y podrían plausiblemente ser presentados como una ideología nacional pan-singapurense" (Englehart 2000, p. 558). Es decir, no se traicionaba la idea de un Singapur para todos los singapurenses, necesaria para la estabilidad nacional.
\end{abstract}

El PAP argumentaba que el paternalismo oficial era algo normal y saludable en las culturas asiáticas, estableciendo que los "asiáticos" deseaban ser gobernados por esta clase de élite. Pero la élite meritocrática resulta desigual porque entre gobernantes y gobernados se establece una jerarquía entre los derechos y obligaciones que teóricamente debían ser recíprocos: por el lado de los gobernantes asegurar el bienestar general y por parte de los gobernados el respeto y la obediencia (idem, p. 553). Desde esta interpretación, al confucianismo se le confirió un rasgo instrumental, ya que los fines esenciales de la incorporación de los seleccionados valores confucianos a la vida pública fueron el mantenimiento del régimen y la continuación de los planes de desarrollo económico (Alatas 2002, p. 217).

Sin embargo, la retórica oficial no podía eliminar un elemento objetivo resultante de la naturaleza del sistema político: su tendencia autoritaria y el temor que el proyecto de ideología nacional despertó entre los sectores de la oposición partidista. Para estos grupos fue evidente el proceso de perfeccionamiento de los sistemas de control político del PAP: los valores de la "sociedad sobre el individuo" y el "consenso en lugar del conflicto" provocaron una fuerte crítica por parte de los dos partidos de oposición en el Parlamento de Singapur porque temían que fueran usados para justificar abusos en los derechos humanos de la población y porque podían usarse para reprimir a la oposición política organizada (Englehart 2000, p. 553). Con el tiempo estos temores se cumplieron puntualmente.

\title{
IV. El discurso occidentalista de la Escuela de Singapur de los valores asiáticos
}

Singapur ha necesitado articular una ideología nacional que lo haga superar su pasado colonial y, al mismo tiempo, lo ayude a dejar de ser un Estado cuya razón de ser sea únicamente su inserción económica en el sistema internacional. Paradójicamente la alternativa que la élite gobernante de Singapur eligió para conformar dicha ideología nacional buscó relacionarse con un movimiento intelectual de alcance internacional con fuerte resonancia en Asia desde los años ochenta y noventa: el debate sobre los valores asiáticos como una fuente explicativa del éxito económico de la región y su contraposición a los valores occidentales.

Como se verá a continuación, el régimen de Singapur trató de inventar su ser nacional más allá de su propio contexto al incorporarse a un fenómeno discursivo producto de los procesos históricos por los que ha atravesado la región de Asia del Este, y el Sureste de Asia en particular, tras el proceso de descolonización de los años cincuenta y la Guerra Fría. 
De esta misma forma, Singapur buscó seguir un proceso de internacionalización reivindicando un conjunto de valores denominados como "asiáticos" por el discurso oficial, los cuales idealmente son compartidos por toda Asia, pero al mismo tiempo reclamando su propia particularidad y su soberanía como Estado. Esto generó una correspondencia entre el proyecto nacional de Singapur y el fenómeno del resurgimiento del confucianismo en la región. De la misma manera, puede considerarse que la eliminación de la confrontación ideológica entre el sistema capitalista y el socialismo realmente existente, en tanto una alternativa, contribuyó a la conformación de un discurso sobre los "valores asiáticos" en los años noventa.

De acuerdo a Gilbert Rozman el debate sobre el resurgimiento del confucianismo en Asia durante las últimas décadas se ha producido en el siguiente contexto. (i) Por un sentimiento de vacío espiritual en las sociedades de Asia que exige una reconciliación entre el individuo y la comunidad; (ii) por la aparición de nacionalismos en la región de Asia del Este y la necesidad, por parte de ciertos regímenes, por justificar sus sistemas políticos a través de sus tradiciones; (iii) por la resistencia a la globalización y a la difusión de los valores culturales occidentales en una región dinámica, en la que se pensaba que el éxito económico debería ser respaldado por el éxito de su cultura (idea que se ha reafirmado con el tiempo, especialmente en China); (iv) como una respuesta a la creciente integración regional en Asia para establecer un regionalismo más estable en el contexto de la globalización (mediante organizaciones internacionales como la Asociación de Naciones del Sureste de Asia (ASEAN), el grupo ASEAN +3, la Cumbre de Asia del Este o el Encuentro Asia Europa) (Rozman 2003, pp. 183-190).

Al atravesar por una condición poscolonial, Singapur continuó luchando por rechazar aspectos de su pasado con el fin de alcanzar una conciencia nacional propia a partir de los proyectos de sus élites. Con el fin de reforzar su proyecto de ideología nacional, el régimen del PAP conformó un movimiento ideológico que desde su aparición fue conocido como la Escuela de Singapur de los valores asiáticos. El debate alrededor de la Escuela de Singapur se convirtió en un intercambio de ideas diverso y se articuló por el conjunto de declaraciones e intercambios tanto escritos como orales sobre los temas que compartían alguna relevancia alrededor del asunto de los valores asiáticos: su existencia, su contenido, y la pertinencia de sus postulados en las dos cuestiones primordiales que aborda el discurso es decir, las políticas públicas y el comportamiento social. "El debate sobre los valores asiáticos no es una discusión oral organizada entre dos partes lanzando respuestas encontradas sobre una misma cuestión" (Emmerson 1995, p. 16).

Algunos de los principales participantes en la llamada Escuela de Singapur de los valores asiáticos eran Lee Kuan Yew y un conjunto de funcionarios e intelectuales orgánicos pertenecientes al PAP entre los que se encontraban servidores públicos, periodistas, académicos y diplomáticos como Kishore Mahbubani, Tommy Koh, Bilahari Kausikan o Wong Kan Seng.

Como se ha comentado, la alternativa elegida por la élite gobernante de Singapur para conformar una ideología nacional se situó en un movimiento intelectual que buscaría tener resonancia en toda la región de Asia del Este, con el fin de llevar a cabo una 'asianización' que legitimara dicha ideología, es decir, transformando a Singapur en un epítome - aparente - de dicha región.

El éxito económico de una nación dota a sus ciudadanos de un sentido de identificación nacional, sin embargo lo económico por sí mismo es insuficiente para la formación de una identidad. Esto fue obvio para el régimen del PAP durante un periodo de recesión económica durante los años ochenta, tiempo en que 
la efectividad del partido en el poder comenzó a ser fuertemente cuestionada por el electorado al mismo tiempo que surgía una creciente oposición partidista.

De la misma manera, durante los años ochenta comenzaban a renacer las tensiones étnicas en Singapur, esta vez producidas por la competencia por los recursos públicos y el desempleo en época de crisis; lo anterior se vio agudizado por un nuevo flujo de trabajadores migrantes (filipinos, malayos e indios) que habían llegado a Singapur en los años anteriores. Además, junto a los cambios demográficos, en el país se observaban nuevas actitudes y comportamientos entre las generaciones jóvenes, más preocupadas por el éxito individual mientras imitaban, a juicio del régimen, la forma de ser de los occidentales, aunque esta crítica en realidad resultaba ser un prejuicio elaborado para llevar a cabo el proyecto de la ideología nacional.

El régimen del PAP decidió que la causa de los problemas anteriores se debía a la influencia "corrosiva" (hedonista, individualista, antisocial) de la cultura occidental sobre Singapur, como una consecuencia negativa de la modernización económica, pero sin cuestionar los fundamentos del sistema económico capitalista. "El resultado fue el incremento numérico de los grupos cívicos que abogaban por una mayor participación política así como de una crítica creciente al estilo autoritario del régimen" (Chi 2003, p. 65).

Los buenos resultados económicos eran insuficientes para detener el proceso de cambio social que exigía una mayor apertura democrática y cuestionaba la legitimidad del régimen; para enfrentarlo el gobierno cambió las reglas en su relación con la sociedad. "En respuesta a la creciente politización, el Estado se orientó a promover y reinventar la ideología del comunitarismo asiático o de los valores asiáticos. Ello se reforzó con la idea de la existencia de una sociedad multicultural existente en "Asia"” (ibidem). Mediante la reinvención del país, los líderes trataban de convertir a Singapur en un centro de negocios, tecnología y asistencia médica para toda la región. "Asia fue insertada en los planes y metas nacionales del país" (ibidem).

Para Alan Chong el discurso de la Escuela de Singapur basaba su legitimidad en los logros del "modelo de desarrollo de Singapur" el cual resultaba excepcionalista al reivindicar una etiqueta como "no occidental en su origen". Sin embargo, la confrontación con Occidente buscaba no sobrepasar el límite de los intereses económicos del país; el discurso resultaba:

"selectivo en su disconformidad hacia la normas liberales que hegemonizaban el orden internacional de la Posguerra Fría. Todo esto se lograría sin dañar los amplios vínculos económicos y militares con los Estados Unidos y la Unión Europea, que se habían desarrollado desde la década de 1980" (Chong 2004, p. 103).

Singapur inició la internacionalización de un discurso poscolonial occidentalista con el fin de evitar la interferencia de lo que consideraba como "valores occidentales" (los cuales también pueden ser la democracia, el libre activismo de los partidos de oposición o la libertad de expresión) en el desempeño del sistema político. Al mismo tiempo, tal internacionalización trataba de identificar las condiciones de la diversidad étnica de Singapur y sus problemas con toda la región del Sureste de Asia y así prevenir la aparición de conflictos. Pero en esta ocasión, usando una interpretación instrumental de Occidente y de Asia con el fin de mantener la estabilidad del régimen político. Como menciona Denny Roy sobre el carácter occidentalista del discurso de los valores asiáticos:

"Asia ha invertido los papeles formando un Otro a partir de Occidente, frente al cual Asia se contrasta favorablemente en laboriosidad, piedad filial y castidad, además de que realiza una crítica moral a las supuestas características de los occidentales" (Roy 1994, p. 232). 
De acuerdo a la interpretación anterior, como los valores occidentales y sus instituciones resultan diferentes a las asiáticas, dichas instituciones no son necesariamente apropiadas para los países de la región.

La inserción de “Asia” en el discurso político nacional agregó una base ideológica con la cual Singapur pudo modificar favorablemente sus relaciones con la región: el reforzamiento de estas relaciones (en lo cual tuvo un papel activo la población de hombres de negocios chinos diseminados en Malasia e Indonesia) fue vista como una condición necesaria para llevar a cabo la redefinición del nacionalismo de Singapur. En el ámbito de las relaciones con las grandes potencias, mediante el discurso de los valores asiáticos el régimen del PAP reclamaba una mayor igualdad política con Occidente, especialmente dadas las tendencias unipolares de Estados Unidos en el contexto de la Posguerra Fría.

"Los diplomáticos de Singapur insistían en este tema sin excepción en sus contribuciones al debate sobre los valores asiáticos. De cierta manera, no es de extrañar que la "Escuela de Singapur" terminara representando una "otredad" a la elite en política exterior de Estados Unidos” (Chong 2004, p. 103).

Igualmente la aparición de la Escuela de Singapur se contextualizó en lo que (aparentemente) se anunciaba como un futuro cambio en la distribución del poder mundial: dicho discurso comenzó a insinuar una alianza retórica entre Singapur y el resto de Asia (incluyendo a China) sobre la legitimidad de un modelo sínico con criterios propios sobre los conceptos de democracia y los derechos humanos. Tal perspectiva iba más lejos, hasta el punto de sugerir una división ideológica global (Latif 1996, p. 322).

Sin embargo, como menciona Chua Beng Huat, resultaba irónico que Singapur, la nación más pequeña de Asia, se presentara a sí misma como una representación del continente más poblado del mundo y sus líderes políticos asumieran la defensa de los valores "asiáticos" más esenciales. No era Asía la que necesitaba de Singapur sino que Singapur necesitaba (y necesita) de Asia, dada la asianización del proceso de formación de su ideología nacional, la cual fue identificada con unos "valores asiáticos" seleccionados por el régimen del PAP (Chua 1993, p. 23).

\section{IV.1. La modernización de las tradiciones: una alternativa de identidad nacional}

Singapur, como otros Estados descolonizados en circunstancias similares, fue creado a través de las actividades de los miembros de una élite educada que buscaba la modernización del país al mismo tiempo que tenía un conjunto de ideas concretas sobre cómo llevar a cabo su particular proyecto de nación. Dicho proyecto podría basarse en lo que previamente consideraba como sus "tradiciones" formativas o referenciales: el concepto de "tradición" de la élite forma parte de su retórica discursiva.

El abanderamiento de una idea oficial sobre las tradiciones del país resulta trascendental, pues de esta forma se construye un diálogo que legitima el proyecto de modernización de los líderes, ya que tal proyecto resulta coherente con el concepto oficial de lo que es la "tradición". Sin embargo,

"todas las tradiciones son inventadas. Ninguna sociedad tradicional fue totalmente tradicional, y las tradiciones y costumbres han sido inventadas por varias razones. No debemos pensar que la construcción consciente de una tradición se da sólo en la sociedad moderna. Es más, las tradiciones siempre llevan incorporadas poder, estén o no construidas de manera deliberada. La tradición se convierte en un mapa a seguir en los procesos socio políticos ya que sirven para estructurar el tipo de relaciones de poder existentes" (Giddens 2000, pp. 53-54). 
Lo relevante en el caso de Singapur es que una tradición puede dejar de ser propiamente tradicional o "destradicionalizarse" para aparecer bajo otras formas y así cumplir con un fin específico. La tensión entre tradición y modernización no es una necesidad, más bien obedece a pugnas por el poder en contextos determinados;

"las tradiciones pueden defenderse de una manera no tradicional - y ese debería
ser su futuro. El ritual, el ceremonial y la repetición tienen unos importantes
papeles sociales, algo comprendidos y respetados por la mayoría de las organi-
zaciones, incluidos los gobiernos. Las tradiciones seguirán sustentándose en la
medida en que puedan justificarse efectivamente, no en términos de sus propios
rituales internos, sino en relación con otros usos y formas de hacer las cosas"
(idem, pp. 57-58).

La invención de las tradiciones parece ser un hecho natural en los procesos de modernización y de construcción nacional. En el caso de Singapur, un grupo de políticos con ambiciones intelectuales, encabezados por Lee Kuan Yew, han usado la formulación de tradiciones desde el poder para dominar la interpretación pública del universo simbólico de la sociedad: el pasado histórico del grupo étnico más numeroso del país, de origen chino, ha ofrecido el confucianismo (su tradición cultural) para articular las orientaciones valorativas y simbólicas que una vez seleccionadas e interpretadas son utilizadas para el resurgimiento, revisión, e invención de la tradición modernizada (Riegel 2000, p. 80).

Según Riegel (idem, p. 79), es común que durante los procesos de modernización se den estos actos que pretenden una reinvención de las tradiciones, lo cual produce un poderoso mecanismo de manipulación sobre la población, ya que estas tradiciones representarán con el paso del tiempo una construcción social cuya realidad será indisputable. Como base material de estos ideales se hallan ciertos proyectos de políticas públicas como los Valores Compartidos; estas políticas son generadoras de las instituciones que sirven como un regulador que asiste a la formulación de la ideología nacional. Tal discurso ideológico se auto realiza una vez que las políticas inciden en un cambio social efectivo (Dirlik 1999, p. 181).

"En tanto que la articulación explícita de los valores asiáticos comunitaristas posee un esencialismo, esta es obviamente una reinvención cultural de un pasado para enmarcar el presente. Sería ingenuo pensar que los progenitores de varias formulaciones sean inconscientes de las construcciones e invenciones que tienen en sus propias manos" (Chua 1998, pp. 4-5).

\section{IV.2. La condición poscolonial de Singapur y los Valores Compartidos}

De acuerdo a Geoffrey Benjamín los políticos de Estados como Singapur, por creerse los depositarios de la inteligencia nacional, se consideran obligados a gobernar a la población en una trayectoria que solamente ellos pueden definir. Estos nuevos Estados nacionales tienden, por consecuencia, a estar orientados permanentemente hacia un futuro imaginado y a una transitoriedad permanente hasta que las élites de gobierno hallen una conformidad con la conciencia social que desean o imaginan exhibir (Benjamin 1988, p. 5).

La herencia de la colonización dio a Singapur las características de una sociedad híbrida en tanto era conformada por diferentes grupos étnicos que habían emigrado al país primordialmente por motivos laborales; con el tiempo, los descendientes de estos grupos se encontraron como miembros de un mismo proyecto de nación. Esto quiere decir que la hibridación de una sociedad, propia de la condición poscolonial, se hallaba implícita en Singapur aun antes de su independencia; "si la hibridación cultural y el sincretismo es la marca de la 
poscolonialidad, entonces la esfera cultural en Singapur, podría decirse, resultaba ser poscolonial antes de ser llamada así” (Chua 1995, p. 14).

Como parte de dicha condición poscolonial aparecen políticas que implicaban una reorganización del Estado nacional como un método para escapar del eurocentrismo heredado del orden colonial. El borrar las fronteras de los grupos étnicos existentes en el país mediante la construcción de una identidad homogénea, aun basada en una reinterpretación del confucianismo y la tensión del discurso occidentalista, fue la respuesta del régimen ante esta herencia híbrida.

Lo relevante del caso de Singapur es que su intento por construir una identidad se elaboró a partir de la reivindicación de la esencia asiática del país: el confucianismo, adoptado por el régimen para crear un paradigma de la identidad asiática usando como contraste a los "valores occidentales" (ibidem).

De acuerdo a Arif Dirlik:

"El discurso poscolonial de nuestros días, en contraste con los primeros días de los movimientos de liberación, pareciera estar obsesionado con el problema de exorcizar el fantasma euro americano que se ha convertido en parte de un legado global" (Dirlik 1999, p. 183). De esta forma, los interesados en conformar una identidad asiática son Estados que perciben en sus tradiciones no solo un sentido de auto identificación sino también un medio para controlar los desajustes sociales provocados por el éxito económico pero que han promovido al capitalismo sin cuestionarlo (ibidem).

La alerta unilateral por parte del régimen del PAP sobre la influencia occidental provocó que diversas voces en el interior de Singapur reaccionaran con cautela e incluso crítica hacia tales declaraciones. Entre los críticos se encontraba un amplio segmento de la población china de habla inglesa y miembros del mismo régimen, quienes argumentaban que tales "valores asiáticos" en realidad eran una reexaminación de un conjunto de supersticiones, tradiciones opresivas y ficciones fuera de época y que en realidad el contenido ético de dichos valores resultaba universal, sin distinción de un credo en particular (como la valorización del trabajo arduo y disciplinado, la educación, la orientación familiar y el colectivismo). Estos valores resultaban necesarios en un sistema de producción como el capitalista, pero el régimen recurriría a ellos para dar una versión esencialista del éxito económico (Chua 1995, pp. 15-16).

"El único valor confuciano el cual es difícil de equiparar convenientemente con alguno de los valores de la burguesía occidental es el de "colectivismo", el cual es propiamente un concepto del confucianismo” (Chua 1998, p. 1).

De acuerdo a Riegel el debate sobre los valores asiáticos se ha dado no solo en el contexto del debate sobre las dinámicas capitalistas en Oriente, sino también a la luz de un debate entre "políticos con ambiciones intelectuales e intelectuales con ambiciones políticas". Esto produce un flujo discursivo continuo que ofrece productos diversos como el "capitalismo confuciano", la Escuela de Singapur y el trasfondo autoritario de su régimen. Estos procesos generan un campo movedizo entre la formulación de una variedad de discursos ideológicos y un análisis objetivo sobre el fenómeno de la reivindicación de los valores asiáticos en la región (Riegel 2000, p. 76).

Particularmente para Lee Kuan Yew, el concepto tradicional confuciano sobre la importancia de la educación ha servido como un capital cultural que impulsa al sector educado de la población de Singapur y así enfrentar los retos de la modernización (Lee 1998, p. 111).

Desde esta interpretación, puede considerarse que Lee Kuan Yew se concibió a sí mismo, y así ha tratado de ser percibido desde el exterior, como una versión moderna del soberano Junzi? de la tradición confuciana cuyo razonamiento ético, idealmente, dirigía e influía los pensamientos y hábitos de sus 
${ }^{14}$ Previamente a la Segunda Guerra Mundial solo algunos antropólogos alemanes habían tratado de dilucidar algún tipo de unidad en el área descrita por ellos como "Sureste de Asia". En realidad dicho término se deriva de la creación del Comando del Sureste de Asia, durante la Segunda Guerra. Posteriormente el término resultó ser útil para definir a una de las regiones estratégicas durante el enfrentamiento bipolar de la Guerra Fría. Con el fin de la Guerra Fría, la validez del término "Sureste de Asia" ha sido sometido a una variedad de críticas (Miller 2004, p. 3). funcionarios; estos últimos, en otro nivel de la jerarquía burocrática, han estado obligados a trasmitir sus instrucciones a los súbditos.

La base ética del proyecto de Valores Compartidos se materializó en un Estado armonizado en el que idealmente prevalece el consenso y en el que las libertades no devienen en anarquía como en Occidente. Sin embargo, de esta manera se produce una cosificación de lo asiático, lo cual provoca que difícilmente se pueda discernir entre el occidentalismo del orientalismo. Las reivindicaciones sobre la superioridad moral o espiritual de Asia sobre el materialismo de Occidente es una posición que niega la historia de Asia, igualmente en un modo orientalista (Dirlik 1999, p. 167).

Por otra parte, debe considerarse que la búsqueda de una identidad nacional entre las unidades políticas de Asia no ha sido una cuestión reciente, si bien a partir de los años noventa el fenómeno se encontró determinado por el contexto de la globalización, el fin de la bipolaridad y el nuevo paradigma histórico que supuso la emergencia de las economías capitalistas de la región (China incluida): este problema surge con el inicio de la dominación occidental en Asia a partir del siglo XVIII (idem, p. 174). De la misma manera, la ubicación geográfica de estos discursos obedece a un patrón similar en el contacto asiático-europeo durante los últimos dos siglos (contacto que, como sabemos, alteró irremediablemente el devenir histórico y cultural de la región). No es coincidencia que el resurgimiento del confucianismo se haya dado en espacios geográficos que fueron en primer término nociones de la especialización orientalista de Asia, realizada por una imaginación eurocéntrica. Como se sabe estas nociones sirvieron a los intereses estratégicos de Estados Unidos durante la Guerra Fría mediante la elaboración de un discurso orientalista utilitario tal como lo ha sido concepto geopolítico de "Sureste de Asia"14.

El debate sobre la identidad ha sido acompañado, en lo general, por el esfuerzo de las sociedades asiáticas por igualar la superioridad tecnológica de los occidentales y mantener una alta efectividad en su desempeño económico (algo que puede remontarse a los proyectos de modernización que trataron de llevar a cabo países como China o Japón desde finales del siglo XIX). Al mismo tiempo, estas zonas eran reformuladas por intereses externos, sin tomar en cuenta la lógica histórica de sus relaciones locales y formaciones culturales; la historia de los flujos migratorios a Singapur es otro ejemplo de ello (idem, p. 177).

Como menciona Yao Souchou, en su forma más exuberante, el capitalismo confuciano aspira a ver más allá de lo históricamente real: "para el Estado de Singapur, sus proyectos serían imposibles sin esta "historia imaginada", la cual alimenta la explicación de su actuación económica en el presente" (Yao 2002, p. 39).

\section{Conclusiones}

Durante los años ochenta el régimen del Partido de Acción Popular de Singapur había alcanzado varios de sus objetivos de desarrollo, consistentes en la planeación de grandes obras de infraestructura, la transformación exitosa de Singapur en un centro confiable para la inversión extranjera y la formulación de políticas públicas efectivas que tuvieran la capacidad de dotar a la población de niveles de vida cada vez más altos. El grado de bienestar social alcanzado a finales durante esa década propició que la élite de gobierno del PAP dirigiera uno de los procesos socio político más complejos que puede realizar un país: aspirar a reinventarse a sí mismo como nación contando para ello con el consenso aprobatorio de la mayoría de la población. La lógica seguida por el discurso oficial para justificar la instrumentación de su proyecto de ideología nacional 
fue que el régimen del PAP había alcanzado un alto grado de legitimidad mediante el efectivo desempeño de los proyectos económicos y de desarrollo.

Los valores de la nación de acuerdo al discurso oficial del PAP se articularían a partir de una tradición, en este caso una reinterpretación instrumental del confucianismo, que serviría para rechazar ciertos aspectos "destructivos" de la modernidad occidental, los cuáles deterioraban la estabilidad social que convenía al régimen del PAP. Si los valores universales, como los derechos humanos, podían convertirse en instrumentos para el intervencionismo desde el exterior, el PAP recurrió a unos valores propios considerados como "tradicionales". Sobre dichos valores, sólo los miembros del PAP podrían realizar la exégesis adecuada, los cuales serían contrastados, a su vez, frente a una imagen orientalista de los "valores occidentales".

Las razones que tienen los políticos para llevar a cabo su reinterpretación de las tradiciones suelen ser similares: mediante la idealización de un pasado histórico, en el cual funcionaban dichas tradiciones, se persigue crear las condiciones para una sociedad cohesionada, estable, ordenada y armoniosa que acepta la legitimidad de la autoridad constituida. De la misma forma y debido a los cambios provocados por la modernización, las tradiciones ofrecen estructuras sociales pretendidamente estables. De la manera anterior fue que el régimen del PAP pretendió presentar a sus políticos profesionales como una versión moderna de los Junzi? confucianos.

En el trabajo se ha señalado que el discurso reivindicador del proceso socio-político anterior sería llevado a cabo por la élite gubernamental e intelectual del PAP, denominada en su momento como la Escuela de Singapur. El debate sobre los "valores asiáticos" generado por los miembros de la Escuela de Singapur se benefició en primera instancia del fin de la Guerra Fría para articular una discusión académica y mediática sobre lo que podría significar una nueva división global: la distinción entre los valores occidentales (en realidad algunos preceptos políticos que se podían relacionar con el liberalismo) frente a los "valores asiáticos" legitimados en el éxito económico de países como Singapur, Malasia, Taiwan o Hong Kong. Sin embargo, como se ha señalado, la reinvención de las tradiciones y el discurso occidentalista, en este caso para encontrar una raíz propia a un proceso de modernización en Asia, ha resultado ser un fenómeno propio de la modernidad determinado por la condición poscolonial de Singapur.

Manuel de Jesús Rocha-Pino (rochapino@yahoo.com) es Maestro en Estudios de Asia y África, especialidad China, por El Colegio de México y Candidato a Doctor en Relaciones Internacionales e Integración Europea en la Universidad Autónoma de Barcelona.

\section{Referencias}

Alatas, S. 2002. Religion, Values and Capitalism in Asia. In: C.J.W-L. Wee, ed. Local Cultures and the New Asia: The State, culture and capitalism in Southeast Asia. Singapur: Institute of Southeast Asia Studies.

Allinson, R. 1990. An Overview of the Chinese Mind. In: R. Allinson, ed. Understanding the Chinese Mind: The philosophical roots. Hong Kong: Oxford University Press.

Benjamin, G. 1988. The Unseen Presence: A theory of the Nation-State and its mystifications. Working Paper n. 91. National University of Singapore.

Bo, M. 2004. A Reexaminacion of the Structure and Content of Confucius "Version of the Golden Rule". Philosophy East \& West, 54(2), pp. 218-248.

Chang, J. 2003. Culture, State and Economic Development in Singapore. Journal of Contemporary Asia, 33(1), pp.85-105.

Chew, E.C.T. 1991. The Singaporean National Identity: Its historical evolution and emergence. In: gapore. Oxford: Oxford University Press.

Chi, J.K. 2003. National-Ethnic Identity Negotiation in Malaysia and Singapore: A State-Society interaction perspective. Berkeley Journal of Sociology, 47, pp. 49-75. 
Los valores compartidos: una reinterpretación política del confucianismo en Singapur

Chong, A. 2004. Singaporean Foreign Policy and the Asian Values Debate, 1992-2000: Reflections on an experiment in soft power. The Pacific Review, 17(1), pp. 95-133.

Chua, B.H. 1993. The Changing Shape of Civil Society in Singapore. Commentary: Civil Society, 11(1), pp. 9-14. . 1995. Culture, Multiracialism and National Identity in Singapore. Working Paper n. 125. Singapur: National University of Singapore.

1998. Taking Asia Out of “Asian Values” Discourse. In: Conferencia Globalizing Cultural Studies. Londres.

Confucio. 1979. The Analects. Traducción del chino por D.C. Lau. Londres: Penguin Books.

Dirlik, A. 1999. Culture against History? The politics of East Asian Identity. Development and Society, 28(2), pp. 167-190.

Emmerson, D.K. 1995. Singapore and the “Asian Values” Debate. Journal of Democracy, 6(4), pp. 95-105.

Englehart, N.A. 2000. Rights and Culture in the Asian Values Argument: The rise and fall of Confucian ethics in Singapore. Human Rights Quarterly, 22(2), pp. 548-568.

Fung, Y. 1998. A Short history of Chinese Philosophy. Nueva York: Simon \& Schuster.

Giddens, A. 2000. Un mundo desbocado. Los efectos de la globalización en nuestras vidas. Madrid: Taurus.

Goh, C.T. 1990. Enhance our Value System. Speeches: A Monthly Collection of Ministerial Speeches, 14(16).

Grubel, H.G. 1991. Singapore's Record of Stability. In: E.C.T. Chew, ed. A History of Singapore. Oxford: Oxford University Press.

Hill, M. 1996. The Politics of Nation Building in Singapore. Nueva York: Routledge.

Hsiao, K-C. 1979. A History of Chinese Political Thought. V. I. Princeton: Princeton University Press.

Kuo, E.C.Y. 1996. Confucianism as Political Discourse in Singapore: The case of an incomplete revitalization movement. In: W. Tu, ed. Confucian Traditions in East Asia Modernity. Cambridge, MA: Harvard University Press.

Latif, A. 1996. The Internationalization of Singapore Politics. Southeast Asian Affairs, 1996, pp. 321-336.

Lee, K.Y. 1991. Preserve Cultural Values, Singapore Develops a National Identity. Speeches: A Monthly Collection of Ministerial Speeches, 15(1).

. 1998. From the Third World to First: The Singaporean Story 1965-2000. Memoirs of Lee Kuan Yew. Singapur: Singaporean Press Holdings.

2003. Culture, State and Economic Development in Singapore. Journal of Contemporary Asia, 33(1), pp. 85-105.

Miller, J. 2004. The Roots and Implications of East Asian Regionalism. Ocassional Paper Series. Asia-Pacific Center for Security Studies.

Perry, M.; Kong, L.; Yeoh, B. 1997. Government and Politics. In: M. Perry; P. Martin; B. Yeoh, eds. Singapore. A Developmental City State. Nueva York: John Wiley \& Sons.

Riegel, K-G. 2000. Inventing Asian Traditions: The Controversy between Lee Kuan Yew and Kim Dae Jung. Development and Society, 29(1), pp. 75-96.

Roy, D. 1994. Singapore, China, and the "Soft Authoritarian” Challenge. Asian Survey, 36(3), pp. 231-242.

Rozman, G. 2003. Center-Local Relations. Can Confucianism boost decentralization and regionalism? In: D. Bell; C. Hahm, eds. Confucianism for the Modern World. Cambridge, UK: Cambridge University Press.

Sartori, G. 2005. Parties and Party Systems: A Framework for Analysis. Colchester: ECPR Press Classics.

Schwartz, B. 1985. Confucius: the Vision of the Analects. In: . The World of Thought in Ancient China. Cambridge, MA: Harvard University Press.

Sin, Y.C. 2000. Can Shu Be the One Word that Serves as the Guiding Principle of Caring Actions? Philosophy East \& West, 50(4), pp. 507-524.

Tamney, J. 1996. The Struggle over Singapore's Soul. Western Modernization and Asian Culture. Nueva York-Berlín: Walter de Gruyter \& Co.

Zhang, W-B. 1999. Confucianism and Modernization: Industrialization and Democratization of the Confucian Regions. Nueva York: St Martin Press.

\section{Otras fuentes}

Singapore Government. 1991. Shared Values. Singapur: Singapore Government. 


\section{ABSTRACT}

This research studies the political process conducted by the government of People's Action Party (PAP) of Singapore during the eighties and nineties of the XXth century to conform a project for the national ideology to legitimize the political system formed in that country after its independence. The project for the national ideology was called as "Shared Values" and it has as one of its main references to a version of Chinese Confucianism which has been adapted (and decontextualized) by the regime of Singapore to fulfill some specific purposes, such as maintaining the stability of its political system and the governing elites. The research analyzes how the project for a national ideology was related to the discourse of "Asian values", existing in East Asia, with the aim of consolidating the order, as well as progress and national identity in a country known because its multicultural diversity. As a conclusion this research attempt to explain how the addition of the "Asian values" into the official discourse of the regime of Singapore was due both to the postcolonial condition of this country and an ideological response to the Orientalist ideas that have been generated about the former Asian colonial areas since the nineteenth century. The study of the Asian values discourse in East Asia (in this case, the characteristics acquired in Singapore) during the eighties and nineties may contribute on the research of contemporary historical development of that region.

KEYWORDS: Popular Action Party; Asian values; Confucianism; Junzi; occidentalism. 